\title{
Exploring the magnetic properties of ferrite nanoparticles for the development of rare-earth-free permanent magnet
}

\author{
Alberto López-Ortega, Università degli Studi di Firenze and INSTM, Sesto Fiorentino (Firenze), I-50019 Italy \\ lopezortega.alberto@gmail.com \\ Elisabetta Lottini, Università degli Studi di Firenze and INSTM, Sesto Fiorentino (Firenze), I-50019 Italy \\ elisabetta.lottini@gmail.com \\ Cesar de Julián Fernández CNR-IMEM Parma, I 43124 (Italy) \\ cesar.dejulian@imem.cnr.it \\ Claudio Sangregorio, CNR-ICCOM Firenze and INSTM, , Sesto Fiorentino (Firenze), I-50019 Italy \\ csangregorio@iccom.cnr.it
}

\begin{abstract}
Currently, there is a rising demand for rare-earth elements for a broad range of industrial applications. However, the risk of supply of these strategic materials due to political restrictions, the high environmental impact of their extraction as well as price oscillations have motivated many researchers in finding viable alternatives. We propose nanometric cobalt ferrite (CoxFe3-xO4) as candidate to achieve this goal. On one hand cobalt ferrite, thanks to its large cubic magnetic anisotropy, is a hard magnet. On the other hand, the reduction to the nanoscale may offer unique advantage, which can significantly improve the characteristic of the material.
\end{abstract}

To this aim a family of monodisperse cobalt ferrite nanoparticles (NPs) with average size covering a broad range (from 4 to $60 \mathrm{~nm}$ ) was synthesized by thermal decomposition of metal-organic precursors. Metal precursors, surfactants and synthetic parameters were settled in order to fine tune the particle size while preserving a narrow particle size distribution. The morphological characterization of the nanoparticles revealed an evolution of the particle shape from spheres to octahedrons through cubes for intermediated sizes.
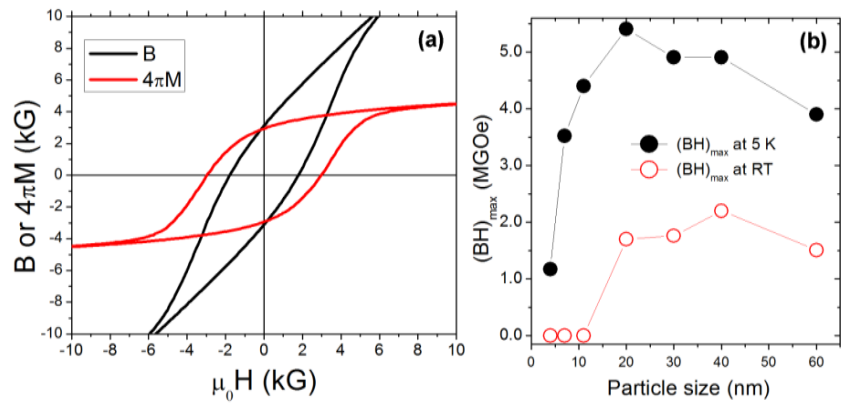

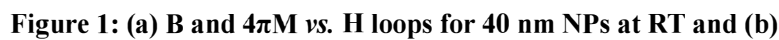
(BH) $\max$ at 5K (black solid circles) and RT (red empty circles) as a function of the particle size.

The dependence of coercive field on particles size displays a non-monotonic behaviour with two distinct maxima values for low and room temperature, respectively (Figure 1). In addition, we evaluated the $(\mathrm{BH}) \mathrm{max}$ product, the figure of merit of permanent magnets, obtaining the maximum value ever reported in the literature for cobalt ferrite NPs (i.e., 2.1 MGOe $(18 \mathrm{kJm}-3)$ for $40 \mathrm{~nm}$ NPs). The collected data gave us the opportunity to discuss the feasibility of the application of this material for the realization of permanent magnet.

Acknowledgement: Research supported by EU-FP7 NANOPYME Project (No. 310516) [www.nanopyme-project.eu]

Keywords-Permanent Magnet, Nanoparticles, Ferrites, Critical materials, Hard magnet, nanomagnetism, synthesis. 